Ann. Biol. anim. Bioch. Biophy's., I975, 15 (3), 559-568.

\title{
ÉTUDE DE LA SURALIMENTATION FORCÉE : EFFETS SUR LE MÉTABOLISME HÉPATIQUE ET LES FORMES DE TRANSPORT HÉPATIQUES CHEZ LE CANARD
}

\author{
B. LECLERCQ et J. C. BLUM \\ avec la collaboration technique de Marie Rose Salichon \\ Station de Recherches avicoles, \\ Centre de Recherches de Tours, I. N.R. A., \\ Nouzilly, 37380 Monnaie, B. P. 1
}

\section{RÉSUMÉ}

Chez le Canard, l'intensité de la lipogenèse hépatique déterminée par l'incorporation du ${ }^{14} \mathrm{C}$-acétate n'est pas augmentée par 4 jours de gavage (ingestion forcée de $300 \mathrm{~g}$ de maïs sec par jour). Mais l'activité de synthèse paraît se maintenir entre chaque gavage, ce qui explique l'accroissement de l'adiposité corporelle. La rétention hépatique mesurée par la décroissance de radioactivité est augmentée par le gavage dans le cas des triglycérides, elle est diminuée dans le cas des phospholipides. L'acide linoléique étant préférentiellement mobilisé dans les lipoprotéines plasmatiques, les triglycérides qui s'accumulent dans le foie en sont très pauvres, ils renferment presque exclusivement des acides gras provenant de synthèses endogènes.

Chez les animaux gavés, on observe une augmentation considérable de la lipémie ; la teneur plasmatique en lipoprotéines de basse densité (VLDL : principale forme de transport des triglycérides) est décuplée. La rapidité du renouvellement, donc du transport, est attestée par une prompte décroissance de la radioactivité des lipoprotéines plasmatiques. Bien que la lipémie des animaux gavés pendant $\mathrm{r}_{3}$ jours demeure constamment élevée, une partie des graisses néoformées est retenue dans l'organe. Le développement du foie gras est donc lié à un transport incomplet des lipides synthétisés in situ.

\section{INTRODUCTION}

La suralimentation forcée des palmipèdes conduit à l'accumulation de triglycérides dans le foie (LECLERCQ et al., I968). Cependant, on ne sait pas si le dépôt de graisse est dû à une hyperactivité du tissu hépatique sans modification de la mobilisation, ou bien s'il y a rétention de lipides par défaut de synthèse des lipoprotéines nécessaires au transport des acides gras hépatiques, comme dans la plupart des stéatoses étudiées chez le Rat. 
I)ans une étude antérieure, nous nous sommes efforcés de répondre à cette question en gavant des poulets et en recherchant l'effet du gavage sur l'incorporation de précurseurs radioactifs dans différents constituants du foie et du plasma. La cinétique d'incorporation de ces précurseurs $\left({ }^{14} \mathrm{C}\right.$-acétate, ${ }^{3} \mathrm{H}$-lysine) n'est guère modifiée, mais on constate que sous l'effet du gavage la teneur du plasma en lipoprotéines VLDL est multipliée par six. Finalement, il semble que la lipogenèse hépatique soit augmentée non pas en intensité, mais en durée par suite d'une lente assimilation digestive des nutriments glucidiques. La mobilisation des triglycérides néoformés sous forme de VLDL explique l'incapacité du Poulet à développer un foie gras volumineux (LeclercQ, Hassan et Blum, I973).

De nouvelles recherches nous ont paru nécessaires pour savoir s'il en était autrement chez les oiseaux choisis habituellement pour la production du foie gras, parce qu'ils peuvent retenir 3 à 5 fois plus de lipides que le Poulet au sein de leur tissu hépatique. L'étude que nous présentons ici concerne deux essais réalisés chez le Canard.

\section{MA'TÉRIEL, E'T MÉTHODES}

\section{Animaux}

Les deux essais portent sur des hybrides de Canard de Barbarie (Cairina moschata) et de Cane de Pékin (Anas platiryncños). Les animaux sont des femelles âgées de I9 semaines.

\section{Protocoles expérimentaux}

\section{Jer essai.}

Deux lots de i 8 canards sont comparés. L'un (lot témoin) reçoit à volonté un aliment complet et équilibré habituellement destiné à cet animal. L'autre est gavé pendant trois jours, à raison de deux repas par jour, avec du mais entier bouilli dans l'eau salée. Les quantités d'aliment ingéré chaque jour sont d'environ $400 \mathrm{~g}$ de matière fraîche, soit $300 \mathrm{~g}$ de matière sèche. Une heure après un repas de $5^{\circ} \mathrm{g}$ pour les animaux du lot témoin et de $200 \mathrm{~g}$ pour ceux du lot gavé, on injecte par voie intraveineuse : $2 \mathrm{ml}$ de solution saline (9 p. Iooo) contenant $20 \mu \mathrm{Ci}$ d'acétate-I-C ${ }^{14}$ de sodium (C. E. A., France; Activité spécifique $45 \mathrm{mCi} / \mathrm{mMole}$ ).

Les animaux sont sacrifiés 30 , 60 et 240 minutes après l'injection de l'isotope par section des veines jugulaires. Le sang est recueilli dans des tubes contenant, sous forme anhydre, un anticoagulant ( I p. Iooo du mélange oxalate de potassium et d'oxalate d'ammonium à parties égales).

Les foies sont prélevés immédiatement, pesés et congelés.

2 essai.

Un lot de ro canards est gavé pendant I 3 jours avec du maïs entier et bouilli dans l'eau salée. Chaque animal ingère en moyenne $600 \mathrm{~g}$ de produit frais au cours des deux repas quotidiens.

Une heure trente après les gavages du matin des $4^{\mathrm{e}}, 8^{\mathrm{e}}$ et $\mathrm{I} 3^{\mathrm{e}}$ jours, on prélève sur oxalate $7 \mathrm{ml}$ de sang par ponction dans la veine alaire. Le plasma est séparé par une centrifugation de Io mn à I ooo g. En fin d'expérience, les animaux sont sacrifiés. Leur foie est pesé.

\section{Techniques d'analyse.}

Après extraction par le mélange chloroforme méthanol $(2-I ; v / v)$, les lipides hépatiques sont séparés en graisses neutres et phospholipides sur acide silicique en utilisant successivement le chloroforme et le méthanol comme éluant. La teneur des graisses neutres en acides gras est déterminée par étalonnage interne grâce à l'acide margarique $\left(C_{17}:{ }_{0}\right)$ en utilisant la chromatographie gaz liquide. 
Pour obtenir les lipoprotéines VLDL, le plasma est centrifugé 60 minutes à 100 ooo g après addition à sa partie supérieure (en évitant tout mélange) de solution saline (9 p. Iooo) selon la technique de COUdert et Raulin (r969). oiseaux.

Cette technique se justifie pour isoler les VLDL du fait de l'absence de chylomicrons chez les

Les lipides de la fraction de très basse densité (VLDL) et ceux ciu plasma entier sont obtenus par extraction dans le mélange chloroforme méthanol $(\mathrm{I}-2 ; \mathrm{v} / \mathrm{v})$; la solution à extraire y étant injectée grâce à une seringue. Par addition d'eau et de chloroforme, la solution est rendue biphasique. Les lipides sont pesés après évaporation du chloroforme. Le dosage des acides gras se fait par étalonnage interne grâce à l'acide margarique.

La radioactivité est mesurée sur les esters méthyliques par scintillation liquide.

\section{RÉSULTATS}

Les résultats du $\mathrm{I}^{\mathrm{er}}$ essai concernant les foies sont présentés dans le tableau $\mathrm{I}$. Chez les animaux témoins, le poids de l'organe et son contenu lipidique tendent à augmenter au cours de l'essai. Les 3 jours de gavage entraînent un accroissement déjà sensible du poids vif des canards. Parallèlement, le poids du foie a presque doublé. Son contenu en lipides est multiplié par un facteur 2 à 3 . L Les graisses neutres rendent compte de la majeure partie de cet accroissement. Fin effet, les acides gras de ces

\section{TABLEAU I}

Infuence d'un gavage de 3 jours sur la composition du foie et l'incorporation de l'acétate $C^{\mathbf{1 4}}$ ( $\mathrm{I} \mathrm{er}$ Essai)

\begin{tabular}{|c|c|c|c|c|c|c|}
\hline \multirow[b]{2}{*}{$\begin{array}{l}\text { Temps écoulé } \\
\text { après l'injection d'acétate }\end{array}$} & \multicolumn{3}{|c|}{$\begin{array}{l}\text { Lot témoin } \\
\text { (Control) }\end{array}$} & \multicolumn{3}{|c|}{$\begin{array}{c}\text { Lot gavé } \\
\text { (Experimental) }\end{array}$} \\
\hline & $300^{\prime}$ & $60^{\prime}$ & $2^{2}+0^{\prime}$ & $30^{\prime}$ & $60^{\prime}$ & $2400^{\prime}$ \\
\hline Poids vif (Body zeeight) $(g)$ & $\begin{array}{r}2428 \\
\pm 54\end{array}$ & $\begin{array}{r}2402 \\
+\quad 123\end{array}$ & $\begin{array}{l}2512 \\
\pm 95\end{array}$ & $\begin{array}{r}2769 \\
\pm 37\end{array}$ & $\begin{array}{r}2707 \\
\pm \quad 100\end{array}$ & $\begin{array}{r}2597 \\
\pm \quad 148\end{array}$ \\
\hline Poids du foie (Liver weight) (g) & $\begin{array}{r}48,8 \\
\pm 1,59\end{array}$ & $\begin{array}{r}54,3 \\
\pm \quad 3,02\end{array}$ & $\begin{array}{r}63,2 \\
\pm \quad 3,29\end{array}$ & $\begin{array}{r}87,3 \\
\pm \quad 17,5\end{array}$ & $\begin{array}{r}81,6 \\
\pm \quad 3,21\end{array}$ & $\begin{array}{r}101,5 \\
\pm \quad 4,05\end{array}$ \\
\hline $\begin{array}{l}\text { Lipides totaux du foie (Total liver } \\
\text { lipids) (mg) }\end{array}$ & $\begin{array}{r}285 \% \\
+\quad 189\end{array}$ & $\begin{array}{r}3278 \\
\pm \quad 317\end{array}$ & $\begin{array}{r}4295 \\
\pm \quad 462\end{array}$ & $\begin{array}{r}11622 \\
\pm \quad 4851\end{array}$ & $\begin{array}{r}8035 \\
+\quad 1136\end{array}$ & $\begin{array}{r}9860 \\
+1375\end{array}$ \\
\hline $\begin{array}{c}\text { Acides gras des graisses neutres } \\
\text { (Neutral fats fatty acids) (mg) }\end{array}$ & $\begin{array}{r}69 \\
+\quad 94\end{array}$ & $\begin{array}{r}586 \\
+\quad 218\end{array}$ & $\begin{array}{r}974 \\
+\quad 131\end{array}$ & $\begin{array}{r}6862 \\
+\quad 3185\end{array}$ & $\begin{array}{r}4202 \\
+\quad 782\end{array}$ & $\begin{array}{r}5678 \\
+\quad 794\end{array}$ \\
\hline $\begin{array}{l}\text { Acides gras des phospholipides } \\
\text { (Phospholipids fatty acids) (mg) }\end{array}$ & $\begin{array}{l}1221 \\
\pm \quad 81\end{array}$ & $\begin{array}{r}1329 \\
\pm \quad 122\end{array}$ & $\begin{array}{r}1717 \\
\pm 253\end{array}$ & $\begin{array}{r}1951 \\
\pm \quad 428\end{array}$ & $\begin{array}{r}1550 \\
\pm 78\end{array}$ & $\begin{array}{r}1707 \\
\pm 226\end{array}$ \\
\hline $\begin{array}{l}\text { Radioactivité totale des acides } \\
\text { gras des G. N. (Neutral fats fatty } \\
\text { acids Total Radioactivity) } \\
\text { d.p.m.) }\end{array}$ & $\begin{array}{r}32013 \\
\pm \quad 810\end{array}$ & $\begin{array}{r}1143 \\
\pm \quad 337\end{array}$ & $\begin{array}{r}434 \\
\pm 92\end{array}$ & $\begin{array}{r}2594 \\
\pm \quad 1 / 1\end{array}$ & $\begin{array}{r}790 \\
\pm 58\end{array}$ & $\begin{array}{r}895 \\
+104\end{array}$ \\
\hline $\begin{array}{l}\text { Radioactivité totale des acides gras } \\
\text { des P. L. (Phospholipids fatty } \\
\text { acids Total Radioactivity) }(1)^{3} \\
\text { d.p.m.) }\end{array}$ & $\begin{array}{r}1414 \\
+\quad 162\end{array}$ & $\begin{array}{r}1666 \\
+\quad 177\end{array}$ & $\begin{array}{r}1133 \\
+\quad 115\end{array}$ & $\begin{array}{r}1175 \\
+\quad 179\end{array}$ & $\begin{array}{r}603 \\
+\quad 57\end{array}$ & $\begin{array}{r}388 \\
+\quad 57\end{array}$ \\
\hline
\end{tabular}


graisses neutres (surtout triglycérides) sont 8 à Io fois plus concentrés dans le foie. Les phospholipides présentent, eux aussi, une augmentation significative mais nettement moins importante. Les radioactivités totales incorporées dans les graisses neutres et les phospholipides sont maximum et sensiblement identiques dans les deux lots 30 minutes après l'injection de l'acétate marqué. Pour les graisses neutres, la décroissance est plus accentuée dans le lot témoin que dans le lot expérimental. On observe le phénomène inverse pour les phospholipides.

TABLEAU 2

Influence d'un gavage de 3 jours sur la composition et le marquage par le $C^{14}$ des lipides plasmatiques ( ${ }^{\mathrm{er}}$ Essai)

\begin{tabular}{|c|c|c|c|c|c|c|}
\hline \multirow{2}{*}{$\begin{array}{l}\text { Temps écoulé } \\
\text { après l'injection d'acétate }\end{array}$} & \multicolumn{3}{|c|}{$\begin{array}{l}\text { Lot témoin } \\
\text { (Control) }\end{array}$} & \multicolumn{3}{|c|}{$\begin{array}{c}\text { Lot gavé } \\
\text { (Experimental) }\end{array}$} \\
\hline & $30^{\prime}$ & $60^{\prime}$ & $240^{\prime}$ & $30^{\prime}$ & $60^{\prime}$ & $240^{\prime}$ \\
\hline Lipémie (Lipemia) (mg/ml) & $\begin{array}{r}5,10 \\
\pm \quad 0,25\end{array}$ & $\begin{array}{r}8,20 \\
\pm \quad 0,99\end{array}$ & $\begin{array}{r}7,56 \\
\pm \quad 0,96\end{array}$ & $\begin{array}{r}10,17 \\
+\quad 0,47\end{array}$ & $\begin{array}{r}9,54 \\
+\quad 0,90\end{array}$ & $\begin{array}{r}12,04 \\
+\quad 0,96\end{array}$ \\
\hline $\begin{array}{r}\text { Acides gras des lipides plasmatiques } \\
(\text { Plasma lipids fatty acids) }(\mathrm{mg} / \mathrm{ml})\end{array}$ & $\begin{array}{r}2,23 \\
+\quad 0,10\end{array}$ & $\begin{array}{r}2,61 \\
\pm \quad 0,33\end{array}$ & $\begin{array}{r}2,02 \\
\pm \quad 0,25\end{array}$ & $\begin{array}{r}4,17 \\
\pm \quad 0,16\end{array}$ & $\begin{array}{r}4,35 \\
\pm \quad 0,42\end{array}$ & $\begin{array}{r}6,30 \\
\pm \quad 0,80\end{array}$ \\
\hline $\begin{array}{l}\text { Acides gras des VLDL (VLDL fatty } \\
\text { acids) }(\mathrm{mg} / \mathrm{ml})\end{array}$ & $\begin{array}{r}0,130 \\
\pm \quad 0,048\end{array}$ & $\begin{array}{r}0,216 \\
\pm \quad 0,074\end{array}$ & $\begin{array}{r}0,168 \\
\pm \quad 0,010\end{array}$ & $\begin{array}{r}1,76 \\
+\quad 0,36\end{array}$ & $\begin{array}{r}2,12 \\
+\quad 0,13\end{array}$ & $\begin{array}{r}2,47 \\
+\quad 0,39\end{array}$ \\
\hline $\begin{array}{l}\text { Radioactivité des } \mathrm{AG} \text { du plasma } \\
\text { (Radioactivity in plasma fatty } \\
\text { acids) (d.p.m./ml) }\end{array}$ & $\begin{array}{r}5908 \\
\pm 1205\end{array}$ & $\begin{array}{r}3895 \\
\pm \quad 365\end{array}$ & $\begin{array}{r}1114 \\
\pm \quad 159\end{array}$ & $\begin{array}{r}7263 \\
+\quad 1160\end{array}$ & $\begin{array}{r}1319 \\
+\quad 186\end{array}$ & $\begin{array}{r}986 \\
+\quad 160\end{array}$ \\
\hline $\begin{array}{l}\text { Radioactivité des AG des VLDL } \\
\text { (Radioactivity in VLDL fatty } \\
\text { acids) }\end{array}$ & $3882^{*}$ & $860^{*}$ & $197 *$ & $\begin{array}{r}4518 \\
\pm 1142\end{array}$ & $\begin{array}{r}806 \\
+\quad 132\end{array}$ & $\begin{array}{r}201 \\
\pm \quad 22\end{array}$ \\
\hline
\end{tabular}

* : Valeurs données à titre indicatif du fait des difficultés de dosage d'aussi faibles quantités d'échantillons.

Dans le tableau 2 sont rassemblés les résultats du I er essai relatifs aux lipides plasmatiques. Chez les animaux témoins, les lipémies varient peu ou tendent seulement à augmenter en fin d'essai. Lre gavage provoque une élévation importante de la lipémie. L'accroissement des VLDL (acides gras des VLDL) est spectaculaire; il rend compte en majeure partie de l'hyperlipémie. En effet, si l'on déduit des acides gras plasmatiques ceux provenant des VLDL, il y a peu de différence entre les lots témoins et gavés. Autrement dit, les lipoprotéines de plus haute densité (HDL + LDL) sont présentes en quantité analogue dans le sang des canards qu'ils soient gavés ou non.

La décroissance de la radioactivité incorporée dans les lipides plasmatiques diffère quelque peu d'un lot à l'autre. Elle est plus importante dans le lot gavé, reflétant la décroissance très rapide de la radioactivité des VLDI. 
Le tableau 3 permet de comparer les compositions en acides gras des diverses classes de lipides séparés au cours du premier essai. Les différences entre les deux lots sont importantes. Dans le foie des canards gavés on constate que les graisses neutres s'appauvrissent considérablement en acide linoléique et dans une moindre mesure en acide stéarique aut profit des acides monoinsaturés. Au sein des phospholipides les différences sont moins prononcées mais là encore, le gavage augmente la proportion d'acides monoinsaturés tout particulièrement d'acide oléique au dépens des acides arachidonique et stéarique. La composition des lipides plasmatiques reflète assez bien celle des graisses neutres du foie. Il en est de même des lipides des lipoprotéines de très basse densité. On constate cependant que dans tous les lipides plasmatiques, l'acide linoléique est nettement plus abondant que dans le foie.

\section{TABLEAU 3}

Composition en acides gras des lipides hépatiques et plasmatiques

\begin{tabular}{|c|c|c|c|c|c|c|c|}
\hline & Lot & $16: 0$ & $16: 1$ & $18: 0$ & $18: 1$ & $18: 2$ & $20: t_{4}$ \\
\hline $\begin{array}{l}\text { Graisses neutres du foie } \\
\text { (Liver neutral fats) }\end{array}$ & $\begin{array}{l}\text { témoin } \\
\text { gavé }\end{array}$ & $\begin{array}{l}36,2 \\
31,3\end{array}$ & $\begin{array}{l}1,2 \\
4,0\end{array}$ & $\begin{array}{l}21.6 \\
13,4\end{array}$ & $\begin{array}{l}27,0 \\
49,9\end{array}$ & $\begin{array}{l}9,2 \\
1,4\end{array}$ & $\underline{3,1}$ \\
\hline $\begin{array}{l}\text { Phospholipides du foie } \\
\text { (Liver phospholipids) }\end{array}$ & $\begin{array}{l}\text { témoin } \\
\text { gavé }\end{array}$ & $\begin{array}{l}28,8 \\
24,4\end{array}$ & $\begin{array}{l}0,8 \\
2,9\end{array}$ & $\begin{array}{l}27,6 \\
23,5\end{array}$ & $\begin{array}{l}12,1 \\
28,3\end{array}$ & $\begin{array}{l}9,3 \\
7,8\end{array}$ & $\begin{array}{l}21,3 \\
11,3\end{array}$ \\
\hline $\begin{array}{c}\text { Acides gras totaux du plasma } \\
\text { (Plasma total fatty acids) }\end{array}$ & $\begin{array}{l}\text { témoin } \\
\text { gavé }\end{array}$ & $\begin{array}{l}28,2 \\
27,0\end{array}$ & $\begin{array}{l}0,9 \\
4,6\end{array}$ & $\begin{array}{l}18,6 \\
13,1\end{array}$ & $\begin{array}{l}26,2 \\
44,0\end{array}$ & $\begin{array}{r}16,4 \\
9,2\end{array}$ & $\begin{array}{l}9,6 \\
2,1\end{array}$ \\
\hline $\begin{array}{l}\text { Acides gras des VLDL } \\
\text { (VLDL fatty acids) }\end{array}$ & $\begin{array}{l}\text { témoin* } \\
\text { gavé }\end{array}$ & $\begin{array}{l}35,5 \\
27,6\end{array}$ & $\begin{array}{l}2,9 \\
3,9\end{array}$ & $\begin{array}{l}20,2 \\
12,2\end{array}$ & $\begin{array}{l}25,5 \\
40,6\end{array}$ & $\begin{array}{l}15,7 \\
10,8\end{array}$ & - \\
\hline
\end{tabular}

* : Valeurs données à titre indicatif du fait des difficultés de dosage d'échantillons aussi faiblement représentés.

Nous présentons dans le tableau 4 les résultats du $2^{\mathrm{e}}$ essai. Au $4^{\mathrm{e}}$ jour de gavage, la lipémie s'élève et atteint un niveau comparable à celle des animaux gavés du $\mathrm{I}^{\text {er }}$ essai (stade 30 minutes $=\mathrm{I}$ heure 30 après le gavage). Il en est de même des VLDL. Par la suite, on assiste à une élévation significative de la teneur du sang en ces deux constituants ; au $8 \mathrm{e}$ jour, la lipémie a augmenté de $2,5 \mathrm{mg} / \mathrm{ml}$ et celle des VLDL de près de $3 \mathrm{mg} / \mathrm{ml}$. Entre le $8^{\mathrm{e}}$ et le $\mathrm{I} 3^{\mathrm{e}}$ jour, la lipémie diminue. L'analyse de variance révèle un effet "individu " toujours significatif. Il correspond à des animaux dont la lipémie ou la teneur du plasma en VLDL est toujours supérieure ou inférieure à la moyenne du lot. Il est cependant impossible de relier significativement l'importance de l'hyperlipémie avec celle de la stéatose. 


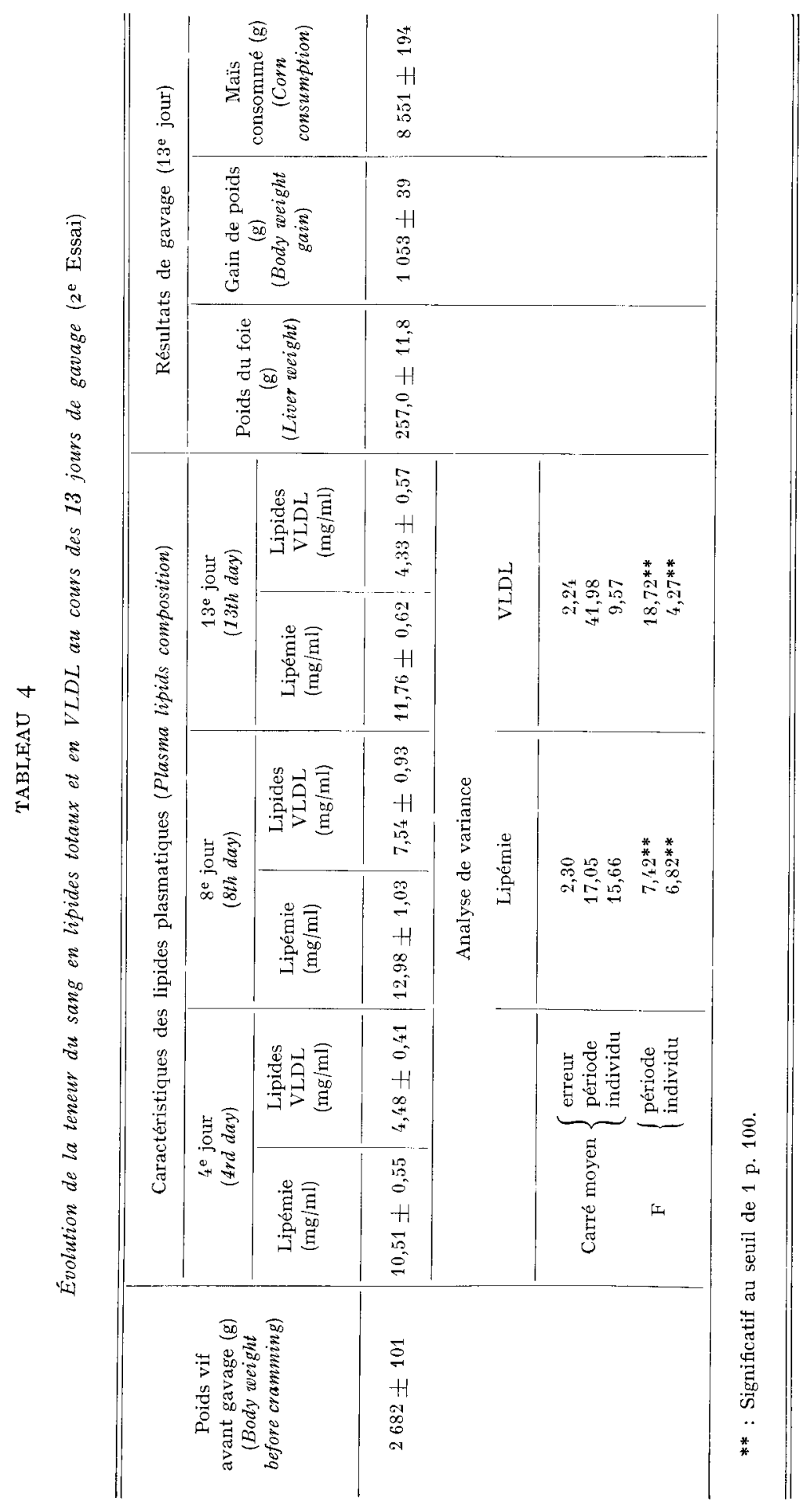




\section{DISCUSSION}

L'ensemble de ces résultats nous permet de rechercher d'une part la signification de 1'hyperlipémie, d'autre part l'origine de la stéatose.

\section{r. - Signification de l'hyperlipémie}

Le gain de poids vif pendant les I 3 jours de gavage (tabl. 4) est de I kilogramme, soit 30 à $5^{\circ} \mathrm{p}$. roo du poids initial. Compte tenu de l'augmentation de poids du foie $(200 \mathrm{~g})$, on peut estimer le gain corporel à $800 \mathrm{~g}$ correspondant essentiellement au développement du tissu adipeux. Or chez les oiseaux, l'activité de synthèse du tissu adipeux paraît réduite (LEVEIL,LE, O'HEA et CHAKRABAR'TY, I968) ; le foie est un lieu de formation privilégié pour les acides gras (O'HEA et LEVEILLE, I969; WAHLE, I974). De ce fait, l'accroissement de la lipémie chez les animaux gavés était prévisible. Parmi les lipoprotéines, les plus légères (VLDI) jouent un rôle privilégié dans le transport des triglycérides du foie vers le tissu adipeux (NELSON, I972; KRUSKI et NARAyaN, I972). Dans notre essai, la teneur plasmatique en acides gras des VLDL est plus que décuplée sous l'effet du gavage (tabl. 2). Chez les canards gavés 4 jours, elle atteint une valeur maximum à la fin de la période d'étude ( 3 heures 30 après le gavage) tandis que chez les témoins, elle tend alors à diminuer. Cela traduit une augmentation permanente du transport des lipides hépatiques vers la périphérie. Cette augmentation est très importante, compte tenu de la vitesse de renouvellement des lipoprotéines VLDL. En effet, nous constatons que la radioactivité des VLDL diminue très vite et qu'on peut considérer que leur durée de vie est aussi brève chez le Canard que chez le Poulet (Kruski et Narayan, I972 ; I ECLERCQ, Hassan et Blum, I974) et chez les mammifères (NELSON, I972). Il apparaît un fait remarquable. La décroissance de la radioactivité des VLDL est aussi rapide chez les animaux gavés que chez les témoins. Cela signifie que dans les deux cas une même fraction de la quantité totale de lipoprotéines sanguines est fixée à chaque instant dans le tissu adipeux. La quantité totale étant décuplée par le gavage, le renouvellement est accéléré d'autant. Ainsi, nous vérifions les résultats obtenus antérieurement chez le Poulet (LECLERCQ, HASSAN et BLUM, I974). Après 4 jours de gavage, l'oiseau réagit comme un mammifère en bonne santé qui disposerait d'une surcharge alimentaire de glucides : le renouvellement des VLDL est accéléré en même temps que s'accroît la teneur plasmatique (NIKkII,A, I969). A ce stade, 1'hypertriglycéridémie reflète la vitesse de transport.

Il peut en être autrement à un stade de gavage ultérieur. I1 apparaît alors des perturbations métaboliques qui permettent d'assimiler les oiseaux gavés à des animaux obèses (BLUM et al., I970). Or chez les animaux obèses, on sait que 1'hypertriglycéridémie (hyperlipémie de type IV ou V) est souvent associée à un ralentissement du renouvellement des lipoprotéines plasmatiques (EATON, I97 I NEIsON, I972). L'obésité correspondant à un état d'embonpoint variable selon les individus, l'hyperlipémie risque de constituer une appréciation de moins en moins précise de l'activité de transport au fur et à mesure qu'avance la période de gavage. Il faut 
aussi tenir compte de tous les facteurs susceptibles d'influencer un paramètre sanguin : genèse des lipoprotéines qui dépend de 1'activité hépatique et dans une moindre mesure de l'activité intestinale (Ruderman et al., I97I ; ANONymE, I974) ; la libération de ces lipoprotéines qui peut être commandée par divers stimuli tels que la digestion, la replétion des cellules hépatiques, l'activité de lipogenèse et l'influence de différentes hormones (NELSON, I972 ; BIERMAN, I972 ; ANONyME, I974). Dès lors, il devient difficile sinon impossible d'apprécier le développement de la stéatose en suivant seulement l'évolution de la lipémie au cours du gavage. Cela explique pourquoi nous n'observons aucune corrélation entre les teneurs plasmatiques en lipoprotéines $\left(4^{\mathrm{e}}, 8^{\mathrm{e}}\right.$ et $13^{\mathrm{e}}$ jours de gavage) et le développement soit du foie gras, soit du tissu adipeux (gain de poids vif).

\section{2. - Origine de la stéatose hépatique}

La quantité totale de graisse synthétisée étant importante, on pouvait s'attendre à un accroissement net de l'activité de la lipogenèse hépatique. Nos résultats montrent qu'il n'en est rien. L'incorporation de l'acétate radioactif est du même ordre chez les animaux gavés pendant 4 jours et chez les animaux témoins (tabl. I). En dépit de cette constatation et de la mobilisation accrue des lipides néoformés, les triglycérides s'accumulent dans le foie sans qu'aucune diminution de la teneur hépatique puisse être observée en cours d'essai I heure 30 à 4 heures après le gavage. Autrement dit, la lipogenèse est maintenue en permanence à un niveau élevé chez les animaux gavés. L'augmentation des synthèses résulte de ce caractère durable. Elle n'est pas liée à un accroissement d'activité. Ce résultat peut être rapproché d'une observation faite par GOODRIDGE (I973) : chez le Poussin nouveau-né c'est 1'utilisation des glucides et non la synthèse des acides gras qui détermine l'intensité de la lipogenèse. Il en est sans doute de même chez 1'adulte. Au cours d'une étude antérieure, nous avions montré que le maïs entier était mieux utilisé pendant le gavage du canard que le maïs sous forme de farine (LECLERCQ et al., I973). La différence semblait liée à une modification de la durée du transit intestinal, le transit trop rapide de la farine ne permettant pas une digestion totale de l'amidon. En définitive, il apparaît possible que la vitesse d'utilisation des glucides alimentaires soit le facteur limitant l'intensité de la lipogenèse hépatique. Des repas, petits ou grands, auraient la même influence, de courte durée pour les premiers (digestion rapide), prolongée dans le temps pour les seconds (assimilation digestive lente).

Les variations de radioactivité des lipides hépatiques permettent d'établir une différence entre les canards gavés 4 jours et les animaux témoins. Le maintien de la radioactivité des acides gras des graisses neutres résulte de la rétention des triglycérides, tandis que la diminution de radioactivité des acides gras des phospholipides montre que ces derniers sont renouvelés rapidement, sans doute parce qu'ils sont mobilisés après incorporation dans les lipoprotéines plasmatiques (OLMSTED, Ig69; NEL,SON, I972). Ainsi, le développement du foie gras est bien lié à une insuffisance relative du système de transport. Malgré son importance, 1'accroissement de la mobilisation des lipides hépatiques vers le tissu adipeux n'assure qu'un transfert incomplet des triglycérides néoformés. Cela oppose le Canard, oiseaul apte à produire de gros foies gras, au Poulet traité dans les mêmes conditions (HASSAN, I973; LECLERCQ, HASSAN et BLUM, I974). 
Ie transport des acides gras hépatiques est d'ailleurs sélectif (tabl. 3). Comme chez les mammifères (SCHONFFis et PFLEGFR, I97I ; Fukazawa, I97I), les acides gras exogènes, en particulier les acides polyinsaturés, sont préférentiellement incorporés dans les lipoprotéines plasmatiques. Une grande part de l'acide linoléique est entraînée par les VLDL, vers la périphérie. Les triglycérides qui s'accumulent dans l'organe proviennent des synthèses réalisées in situ. Nous confirmons des travaux antérieurs réalisés chez 1'Oie (LECLERCQ, I968) à une différence près : chez le Canard, la proportion d'acides gras saturés n'augmente pas, c'est l'acide oléique qui est le plus abondant.

En conclusion, chez les Oiseaux (Poulet, Canard), l'activité de la lipogenèse hépatique dépendrait directement de la vitesse d'utilisation des glucides alimentaires (assimilation digestive, glycolyse). La grande quantité d'aliment ingéré à chaque gavage serait digérée lentement prolongeant dans le temps les synthèses hépatiques sans accroître leur intensité.

Le quatrième jour de gavage, à l'encontre du Poulet (LECLERCQ, HAssan et BLUM, I974), le Canard est capable de retenir in situ une partie des lipides néoformés. Cela rend compte de la différence d'aptitude des deux espèces à la production du foie gras. I1 reste à savoir dans quelle mesure une défaillance du système de transport doit être seule invoquée. La mobilisation des triglycérides vers la périphérie dépend tout autant de la possibilité des hépatocytes à constituer des réserves que des moyens de transport. L'hypertrophie hépatique observée chez l'Oie semble indiquer que, à son début, tout au moins, le développement du foie gras est plus physiologique que pathologique (BLUM et LECLERCQ, I973). La quantité de graisse présente dans la cellule hépatique pourrait exercer une stimulation variable selon les espèces sur la synthèse et la sécrétion des lipoprotéines (NELSON, I972). En définitive, la recherche des facteurs responsables de la formation du foie gras passe par une meilleure connaissance des mécanismes contrôlant le transport des lipides d'origine endogène.

Recu pour publication en avril 1975.

\section{SUMMARY}

FORCE-FEEDING : EFFECTS ON ME'TABOLISM

AND IIPID TRANSPOR'T IN DUCK LIVER

\footnotetext{
When ducks are force-fed $300 \mathrm{~g}$ of dry corn per day for 4 days, intensity of hepatic lipogenesis, determined by incorporation of ${ }^{14} \mathrm{C}$-acetate, does not increase. However, synthesis activity seems to continue between each force-feeding, which would explain the increase of body adiposity. Force-feeding increases hepatic retention of triglycerides, as measured by a decrease in radioactivity; it decreases phospholipid retention. Triglycerides accumulating in the liver are very poor in linoleic acid, which is preferably mobilized in plasma lipoproteins; these triglycerides are almost entirely composed of fatty acids from endogenous syntheses.

Blood lipid content increases in force-fed animals; the amount of low-density plasma lipoprotein (VLDL : main type of triglyceride transport) is ten times greater. Rapid turn-over and thus transport rate, is shown by an immediate decrease in plasma lipoprotein activity. Although blood lipid content of animals force-fed for I 3 days remains constantly high, part of the neoformed fat is retained in the organ. The development of fatty liver is thus related to incomplete transport of lipids synthesized in situ.
} 


\section{RÉFÉRENCES BIBLIOGRAPHIQUES}

Anonyme, 1974. Mechanisms of carbohydrate-induced hypertriglyceridemia. Nutr. Rev., 32, 74-75.

Bierman E. L., T972. Insulin and hypertriglyceridemia. Israël J. Med. Sci., 8, 303-308.

Blum J. C., Gaumeton J. C., Muh J. P., Leclerce B., ig7o. Modifications de la valeur des normes sanguines en fonction du degré de stéatose hépatique chez l'oie gavée. Ann. Rech. Vêt., 1, I67-I78.

Blum J. C., Leclerco B., I973. Nouvelles précisions sur les modifications biochimiques et histologiques du foie provoquées par le gavage. Journées Avicoles de Varèse (Italie), 3I mai-4 juin, 2, I93-207.

Coudert G., Raulin J., I969. Transport sélectif des acides gras par les lipoprotéines plasmatiques du porc au cours du jeûne. Ann. Nutr. Alim., 23, I-29.

EAton R. P., I97I. Synthesis of plasma triglycerides in endogenous hypertriglyceridemia. J. Lipid Res., 12, $49 \mathrm{I}-497$.

Fukazawa T., I97I. Effect of essential fatty acids deficiency on lipid transport from liver. Lipids 6, 388-393.

GOODRIDGE A. G., 1973. On the relationship between fatty acid synthesis and the total activities of acetyl coenzyme $A$ caboxylase and fatty acid synthetase in the liver of prenatal and early postnatal chicks. J. Biol. Chem., 248, 1932-r938.

Hassan I., r973. Influence de divers facteurs alimentaires sur la stéatose hépatique du Poulet suralimenté par gavage. Thèse Doct. Ing., 79 p., Paris.

Kruski A. W., Narayan K. A., 1972. Lipoprotein synthesis in chicken fed cholesterol. Atherosclerosis, 15, I 4I-I 45.

Leclerce B., Durand G., Delpech P., Blum J. C., I968. Note préliminaire sur l'évolution des constituants biochimiques du foie au cours du gavage de l'Oie. Ann. Biol. anim. Biochim. Biophys., 8, $549-556$.

Leclerce B., Hassan I., Blum J. C., I974. The influence of force-feeding on the transport of plasma lipids in the chicken (Gallus gallus L.). Comp. Biochem. Physiol., 47 B, 289-296.

Leclerce B., Hassan I., Thivend P., Blum J. C., I973. Utilisation comparée du maïs entier et du maîs broyé pour le gavage du Canard. Ann. Zootech., 22, 337-34o.

Leveille G. A., O'Hea F. K., Chakrabarty $\mathrm{K}_{\text {, , }}$ 1968. In vivo lipogenesis in the domestic chicken. Proc. Soc. Exp. Biol. Med., 128, 398-40I.

Nelson G. J., I972. Blood lipids and lipoproteins-quantitation, composition and Metabolism. I Vol., 980 p. John Wiley and sons, New-York.

Nikkila E. A., I969. Control of plasma and liver triglyceride kinetics by carbohydrate metabolism and insulin. Ad. Lipids Res., 7, 63-134.

O'Hea E. K., Leveille G. A., r969. Lipid biosynthesis and transport in the domestic chick (Gallus domesticus). Comp. Biochem. Physiol., 30, I49-I59.

Olmsted C. A., i969. Studies on the role of phospholipids in the triglyceride cycle. Lipids, 4, 40I-407.

Ruderman N. B., Jones A. L., Krauss R. M., Shafrir E., 197r. A biochemical and morphologic study of very low density lipoproteins in carbohydrate induced hypertriglyceridemia. J. Clin. Invest., 50, I355-1 368 .

Schonfeld G., PFLEGer B., I97I. Utilization of exogenous free fatty acids for the production of very low density lipoprotein triglyceride by liver of carbohydrate fed rats. J. Lipid Res., 12,6r4-62r.

WAHLE K. W. J., 1974. Desaturation of long-chain fatty acids by tissue preparations of the sheep, rat and chicken, $48 \mathrm{~B}, 87$-105. 